\title{
Reliability Analysis of Residential Photovoltaic Systems
}

\author{
A. Garro ${ }^{1}$ and F. Barrara \\ ${ }^{1}$ Department of Electronics, Computer and System Sciences \\ D.E.I.S., University of Calabria \\ Campus of Arcavacata, Via P. Bucci 41C, 87036 Rende (Italy) \\ Phone/Fax number:+0039 0984 494713, e-mail: alfredo.garro@unical.it, f.barrara@libero.it
}

\begin{abstract}
Due to various incentive programs and local market conditions of several European and worldwide countries, photovoltaic systems are rapidly becoming the most diffused solution for residential installations. This wide adoption raises new and important issues related to the efficiency, efficacy and safety of these highly distributed and heterogeneous power systems. In particular, as these systems are often connected to the general power grid so that a system failure may affect the operation of other interconnected systems, in their design an accurate dependability analysis should be performed. Moreover, a dependability analysis allows establishing a maintenance plan acceptable to the user for keeping the system operating effectively. However, despite these reasons, this kind of analysis is often missing manly due to the lack of specific methodological approaches and related ready-to-use tools. To overcome this lack, the paper presents a methodological approach to the reliability analysis of residential photovoltaic systems which exploits analysis techniques and models coming from the R.AM.S. (Reliability, Availability, Maintainability, and Safety) engineering discipline.
\end{abstract}

\section{Key words}

Photovoltaic Systems, Dependability Analysis, Reliability Block Diagrams, Software Environments for Reliability Analysis.

\section{Introduction}

The rapid climatic change which is characterizing the last decades is strongly related to the impact of human activities on the environment and, in particular, to the carbon dioxide $\left(\mathrm{CO}_{2}\right)$ emissions produced from fossil fuels [2]. A real reduction of the $\mathrm{CO}_{2}$ emissions can be manly obtained by drastically changing the energy generation mix: from the currently predominant fossil fuelled energy mix to a nuclear and renewable energy (solar, wind, biofuels, hydropower, geothermal) mix [1, 8].

In particular, also due to various incentive programs and local market conditions [6], in several countries as France, Italy, Germany, Japan, South Korea, and the U.S, the number of commercial, industrial and residential solar power systems installations (Photovoltaic or Concentrated Solar Power Systems) is rapidly increasing. The wide adoption of solar power systems raises new and important issues related to the efficiency, efficacy and safety of these highly distributed and heterogeneous power systems which are often connected to the general power grid so that a system failure may affect the operation of other interconnected systems. However, in the design of solar power system, and especially in that of residential photovoltaic systems, a quantitative and full-fledged analysis of the system dependability is often missing manly due to the lack of specific methodological approaches and related ready-to-use tools [3, 7]. In this context, the paper presents a methodological approach which aims to apply analysis techniques and models coming from the R.A.M.S. (Reliability, Availability, Maintainability and Safety) engineering discipline to the reliability analysis of residential photovoltaic systems. Specifically, the approach is centered on a series-parallel system reliability analysis which allows to evaluate the reliability of a typical residential photovoltaic system given its architecture, the characteristics of the exploited components, and the reference time horizon. Moreover, a Java-based application which implements the proposed reliability analysis has been developed so to guide the user, in a step by step way, from the system architecture definition to the generation of the documentation which clearly reports the analysis results.

The rest of the paper is structured as follow: Section 2 introduces the R.A.M.S. analysis discipline along with its main methods and techniques; Section 3 presents the proposed reliability model for a residential photovoltaic system; Section 4 presents SERA, the Software Environment for Reliability Analysis which implements the proposed model; finally, conclusion are drawn and future work delineated.

\section{R.A.M.S. Analysis: models and techniques}

RAMS is the abbreviation of Reliability, Availability, Maintainability and Safety and indicates an engineering discipline which aims at providing an integrated and methodological approach to deal with system dependability [4]. In particular, Reliability represents the ability of a system to perform its required functions under stated conditions, identified during its design, for a specified period of time; Availability is the proportion of 
time a system is in a functioning condition defined at design time; Maintainability represents the ease with which maintenance of a system can be performed in accordance with prescribed requirements; Safety takes into account the effects of the system on its surrounding environment to prevent, eliminate and control hazards.

The main objective of the RAMS analysis is then to identify the causes of failure of a system and its components. Specifically, the concept of time-to-failure is modeled as a random variable $\boldsymbol{T}$ and by the following related function:

1) Reliability function, which indicates the probability that the system fails after time $t$ :

$$
R(t)=\operatorname{Pr}(T>t)
$$

2) Failure distribution, which is a cumulative distribution function and indicates the probability that a system fails before time $t$ : $F(t)=\operatorname{Pr}(T \leq t)=1-R(t)$;

$$
F(t)=\operatorname{Pr}(T \leq t)=1-R(t)
$$

3) Failure density function, which indicates how the probability of failure is distributed over the life of the system:

$$
f(t)=\frac{d}{d t} F(t)=\dot{F}(t)
$$

4) Hazard function (or hazard rate), which indicates the probability of a system failure between $t$ and $t+\Delta t$ given that it was operating at time $t$, and becomes the instantaneous failure rate as $\Delta \mathrm{t}$ tends to zero:

$$
\lambda(t)=\lim _{\Delta t \rightarrow 0} \frac{R(t)-R(t+\Delta t)}{R(t) \cdot \Delta t}=\frac{-\dot{R}(t)}{R(t)}
$$

For a wide range of mechanical systems the function $\lambda(t)$ assumes a typical shape called Bathtub curve which shows three different stages of failures during the system life:

- early failures, the failure rate is high due to the infant mortality of system components but rapidly decreasing;

- random failures, the failure rate is low and constant (useful life of the system);

- wear-out failures, the failure rate increases as age and wear take their toll on the system components.

5) Mean Time To Failure (MTTF), which represents the expected value of the time-tofailure random variable $T$ :

$$
M T T F=E(T)=\int_{0}^{\infty} t f(t) d t
$$

In Table I the expressions of the above introduced functions in terms of each of them are reported.
Table I. - Main Reliability Functions

\begin{tabular}{|c|c|c|c|c|c|}
\hline & $\begin{array}{c}f(t) \\
\text { Failure density } \\
\text { function }\end{array}$ & $\begin{array}{c}F(t) \\
\begin{array}{c}\text { Failure } \\
\text { distribution }\end{array}\end{array}$ & $\begin{array}{c}\boldsymbol{R}(t) \\
\text { Reliability } \\
\text { function }\end{array}$ & $\begin{array}{c}\lambda(t) \\
\text { Hazard function }\end{array}$ & $\begin{array}{c}\text { MTTF } \\
\text { Mean Time to } \\
\text { Failure }\end{array}$ \\
\hline$f(t)$ & $\int_{0}^{\infty} f(\tau) d \tau$ & $\int_{t}^{\infty} f(\tau) d \tau$ & $\frac{f(t)}{1-\int_{0}^{t} f(\tau) d \tau}$ & $\int_{0}^{\infty} t f(t) d t$ \\
\hline$F(t)$ & $\frac{d F(t)}{d t}$ & & $1-F(t)$ & $\frac{d F(t)}{d t} * \frac{1}{1-F(t)}$ & $\int_{0}^{\infty}(1-F(t)) d t$ \\
\hline $\boldsymbol{R}(t)$ & $\frac{-d R(t)}{d t}$ & $1-R(t)$ & & $\frac{-d R(t)}{d t} * \frac{1}{R(t)}$ & $\int_{0}^{\infty} R(t) d t$ \\
\hline$\lambda(t)$ & $\lambda(t) e^{-\int_{0}^{t} \lambda(\tau) d \tau}$ & $1-e^{-\int_{0}^{t} \lambda(\tau) d \tau}$ & $e^{-\int_{0}^{t} \lambda(t) d \tau}$ & & $\int_{0}^{\infty} e^{-\int_{0}^{t} \lambda(\tau) d \tau} d t$ \\
\hline
\end{tabular}

The reliability analysis of a system is typically facing using a layered approach and through quantitative and qualitative analysis techniques [9].

Quantitative analysis techniques are based on the identification and modeling of physical and logical connections among system components and on the analysis of their reliability through statistical methods and techniques. One of the widely adopted techniques is the series-parallel system reliability analysis in which the system is modeled as a combination of blocks of components organized, from the reliability point of view, in a series and/or parallel manner. In particular, in a series model a system is considered to be operating at time $t$ if and only if all its components are properly working at time $t$; whereas, in a parallel model, the system is considered to be operating at time $t$ if at least one of its components is properly working at time $t$. In both models the failure of each component is supposed to be independent of the failure of the others. The reliability of a system modeled as a series of $n$ components with constant failure rates $\left(\lambda_{i}(t)=\lambda_{i}, i=1 . . n\right)$ can be evaluate as follows:

$R_{s}(t)=\prod_{i=1}^{n} R_{i}(t)=\prod_{i=1}^{n} e^{-\lambda_{i}(t) t}=e^{-\sum_{i=1}^{n} \lambda_{i} t}=e^{-\lambda_{\text {sum }} t}$

Whereas, for a system modeled as a parallel of components, reliability assumes the following form:

$$
R_{p}(t)=1-F_{s}(t)=1-\prod_{i=1}^{n}\left(1-R_{i}(t)\right)
$$

By composing series-parallel blocks of components a complete Reliability Block Diagram (RDB) of a system can be obtained and analyzed.

Another popular quantitative analysis technique is based on the exploitation of Markov Chain models in which system reliability is modeled as a discrete random process and dependencies among system components and their working state are represented by means of a directed graph where edges are labeled by the probabilities of going from one state to the other states.

Qualitative Analysis techniques aim to identify the possible system failures, their rate of occurrence and (local and global) effects on the system so to individuate corrective actions. Two main techniques are currently exploited: FMECA (Failure Modes Effects and Critical Analysis) and Fault Tree Analysis.

FMECA aims to assess the effects of possible failures and correlate them to the failure rate of occurrence. This risk assessment provides two steps: (i) preparation of FMECA worksheets, in which critical events (failure modes) are identified and the effects of them on the global system are evaluated following a standard 
worksheet format; (ii) risk priority numbers evaluation, in which the risk associated with each failure mode is assessed by using the formula $R P N=S * O * D$, where $\mathrm{S}$ (Severity) is a numerical subjective estimate of how severe is the effect of a failure mode, $\mathrm{O}$ (Occurrence) is the rate of occurrence of the failure mode, D (Detection) is a numerical subjective estimate of the effectiveness of the controls to prevent or detect the cause or failure mode before the failure happens. The evaluation of RPN leads to the definition of a risk matrix which gives a compact and visual representation of the severity of the risk of the considered critical events.

Fault Tree Analysis is a graphical tool for analyzing failures which after selecting a critical event and analyzing its causes constructs a fault tree in which the root is the selected event (top event) and each contributory event is added to the tree by using logic gates (and, or, xor, etc.). The constructed tree allows identifying critical paths; moreover, when a fault tree is labeled with actual numbers about failure probabilities it can be used for quantitative analysis.

\section{Reliability Modeling of Residential Photovoltaic Systems}

In a Solar Photovoltaic (PV) System the solar radiation is converted into direct current (DC) electricity using semiconductors that exhibit the photovoltaic effect [5]. Three main categories of solar PV systems can be individuated:

- Stand-alone PV systems (or off-grid PV systems), which are not connected to the utility power lines and could either be used to charge batteries that serve as an energy storage device or directly work using the solar energy available in the daytimes.

- Grid-connected PV systems (or on-grid systems), which interact with utility grid so that power can be drawn from/provided to the utility grid on the basis of the balance between the energy produced by the PV system and that required by the load connected to it; these grid connected systems are designed with or without battery storage.

- Hybrid systems, which are systems with more than one source of power as a PV/Wind system where a wind turbine is added to a PV system to provide complementary power generation.

Due to various incentive programs and local market conditions [6], among the three introduced types, in several European countries Grid-connected PV systems are rapidly becoming the most diffused solution for residential installations. In particular, a residential and grid-connected PV system is basically constituted by the following components (see Figure 1):

- PV Generator, which consist of a set of solar panels, mounted on the roof or in open spaces; each panel is a packaged interconnected assembly of solar cells that converts the energy of sunlight directly into DC electricity; panels are interconnected to create arrays with the desired peak DC voltage and current;
- Inverter, that transform the PV generated DC electricity to the grid electricity (which is of $\mathrm{AC})$ at the grid voltage;

- Energy Meter, which measures, after the DC$\mathrm{AC}$ conversion, the energy produced by the PV generator; this meter is introduced for two main purposes: (i) monitoring the performance of the PV system; (ii) calculating the amount of produced energy on the basis of which, in several European countries, a monetary incentive is offered to the owner of the PV system;

- Import/Export Meter, which measures the energy exchanged between the PV system and the utility grid; in fact, the electricity produced during the daytime is either used by the property owner, or directed back into the electricity grid and purchased by a utility company (an arrangement called "net metering"); at night, or on dark days when the panels do not produce sufficient power, electricity will be supplied via normal utility company grid system.

Finally, several Electrical Boxes are installed to connect system components and protect them against short circuits and voltage peaks.

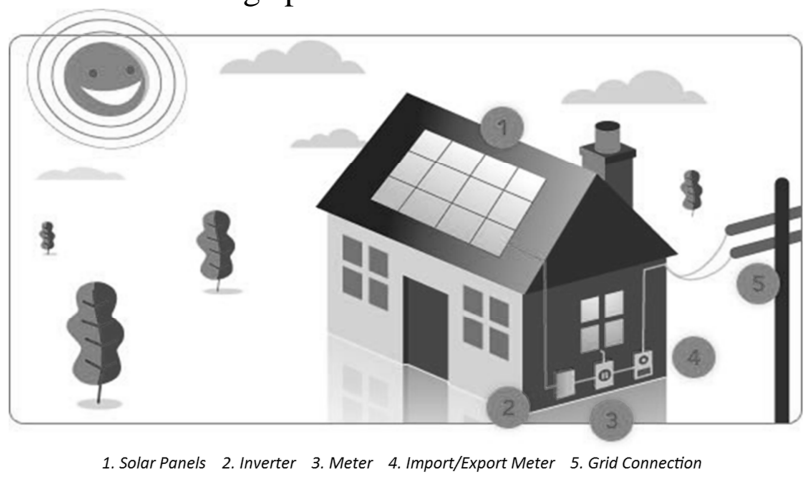

Fig. 1. A typical residential and grid connected PV system

From a reliability point of views, the above mentioned components form a series system as a failure in each of them inevitably produces a failure of the whole PV system. In fact, a PV system is considered properly working not only if it is able to produce a given amount of electricity given certain weather condition, but also if the produced energy can be correctly measured and effectively utilized. Thus, by applying Eq. (6), the reliability of a PV system like that of Figure 1 and with two Electrical Boxes (one after the PV Generator and one before the Import/Export Meter), can be calculated as follows:

$$
\begin{aligned}
& \boldsymbol{R}_{P V \_ \text {System }}(\boldsymbol{t})=\boldsymbol{R}_{P V G e n}(\boldsymbol{t}) * \boldsymbol{R}_{E B o x 1}(\boldsymbol{t}) * \boldsymbol{R}_{I n v}(\boldsymbol{t}) * \boldsymbol{R}_{\text {EMeter }}(\boldsymbol{t}) * \\
& \text { * } \boldsymbol{R}_{E B o x 2}(t) * \boldsymbol{R}_{I / \text { EMeter }}(\boldsymbol{t})
\end{aligned}
$$

The Reliability $R(t)$ of each components can be expressed in function of the component Hazard Rate $\lambda(t)$ (see Table I), thus Eq. (8) becomes:

$$
\begin{aligned}
& \boldsymbol{R}_{P V_{-} S_{y s t e m}}(\boldsymbol{t})=e^{-\int_{0}^{t} \lambda_{P V G e n}(\tau) d \tau} * e^{-\int_{0}^{t} \lambda_{E B o x}(\tau) d \tau} * \\
& * e^{-\int_{0}^{t} \lambda_{I n v}(\tau) d \tau} * e^{-\int_{0}^{t} \lambda_{E M e t e r}(\tau) d \tau} * e^{-\int_{0}^{t} \lambda_{E B o x}(\tau) d \tau} * \\
& * e^{-\int_{0}^{t} \lambda_{I / E M e t e r}(\tau) d \tau}
\end{aligned}
$$


For calculating Eq. (9) the Hazard Rates of the individuated system components has to be evaluated.

For the Inverter, Energy Meter, Import/Export Meter, and Electrical Boxes, during their useful life, the Hazard Rates can be considered constant, thus their Reliability Functions can be evaluated as follows:

$$
\begin{aligned}
& \boldsymbol{R}_{\text {comp }}(\boldsymbol{t})=\boldsymbol{e}^{-\int_{0}^{t} \lambda_{\text {comp }}(t) d \tau}=e^{-\int_{0}^{t} \lambda_{\text {comp }} d \tau}=e^{-\lambda_{\text {comp }} t} \\
& \text { where comp }=\{E B o x 1, \text { Inv, EMeter, EBox 2, I/EMeter }\}
\end{aligned}
$$

Moreover, the Hazard Rate can be obtained as the inverse of the MTTF (Mean Time to Failure) of the component: $\lambda_{\text {comp }}(t)=\lambda_{\text {comp }}=1 / M T T F_{\text {comp }}$ (see the formula which relates $\lambda(t)$ and MTTF in Table I).

The evaluation of the Reliability of the $P V$ Generator $\left(R_{P V G e n}(t)\right)$ requires instead a more elaborate analysis as the Generator is in turn constituted by solar panels interconnected to create arrays with the desired peak DC voltage and current. In fact, a Solar Panel is able to generate under Standard Test Conditions (STC) a peak power $\mathrm{SP}_{\mathrm{PP}}$ (measured in $\mathrm{kW}_{\mathrm{p}}$ ); thus, for obtaining a given peak power from the PV Generator $\left(\mathrm{PVG}_{\mathrm{PP}}\right)$ at least a number $\mathrm{NSP}_{\mathrm{REQ}}=\mathrm{PVG}_{\mathrm{PP}} / \mathrm{SP}_{\mathrm{PP}}$ of Solar Panels must be interconnected. In practice, the total number of interconnected solar panels of the PV Generator $\left(\mathrm{NSP}_{\mathrm{PVG}}\right)$ is typically greater than $\mathrm{NSP}_{\mathrm{REQ}}$ in order to introduce redundancy for increasing the reliability of the generator. Indeed, supposing that a failure of a solar panel does not affect the operation of the others and that the PV Generator correctly operates if at least $\mathrm{NSP}_{\mathrm{REQ}}$ solar panels are properly operating, from a reliability point of view ${ }^{1}$, the array of solar panels which constitutes the Generator can be modeled as a series-parallel system constituted by $\mathrm{NSP}_{\text {SERIES }}=\mathrm{NSP}_{\mathrm{REQ}^{-}}-1$ panels in series followed by $\mathrm{NSP}_{\mathrm{PARALLEL}}=\mathrm{NSP}_{\mathrm{PVG}_{\mathrm{G}}}-\mathrm{NSP}_{\mathrm{REQ}}+1$ panels in parallel.

As an example, if $\mathrm{PVG}_{\mathrm{PP}}=3 \mathrm{~kW}_{\mathrm{p}}$ and $\mathrm{SP}_{\mathrm{PP}}=0,3 \mathrm{~kW}$, a $\mathrm{PV}$ Generator with $\mathrm{NSP}_{\mathrm{PVG}}=12$ panels can be modeled, from a reliability point of view, as a series-parallel system with $\mathrm{NSP}_{\text {SERIES }}=\mathrm{NSP}_{\mathrm{REQ}^{-}}-1=\mathrm{PVG}_{\mathrm{PP}} / \mathrm{SP}_{\mathrm{PP}}-1=9$ panels in series followed by $\mathrm{NSP}_{\mathrm{PARALLEL}}=\mathrm{NSP}_{\mathrm{PVG}^{-}}$ $\mathrm{NSP}_{\mathrm{REQ}}+1=12-10+1=3$ panels in parallel (see Figure 2); in this reliability configuration the $\mathrm{PV}$ Generator is correctly working if at least $\mathrm{NSP}_{\mathrm{REQ}}=10$ panels are properly working.

The introduced reliability model for the PV Generator allows calculating its Reliability function as follows (see Eq. (6) and (7)):

$R_{\text {PVGen }}(t)=e^{-\sum_{i=1}^{N S P_{S E R I E S}} \lambda_{P_{i}} t} *\left[1-\prod_{i=1}^{N S P_{\text {PARALLEL }}}\left(1-e^{-\lambda_{P S_{i}} t}\right)\right]$

In (11) $\lambda_{\mathrm{PS}}$ indicated the hazard rate of a Solar Panel which it is supposed to be constant during its useful life; if all the exploited panels have the same $\lambda_{\mathrm{PS}}$ Eq. (11) becomes:

${ }^{1}$ It is worth recalling that a series-parallel reliability model of a system although it is influenced by the physical connections among the system components does not merely reproduce it.

$$
R_{\text {PVGen }}(t)=e^{-N S P_{S E R I E S} \lambda_{P S} t} *\left[1-\left(1-e^{-\lambda_{P S} t}\right)^{N S P_{P A R A L L E L}}\right]
$$

From Eq. 12, It can be easily demonstrated that although the hazard rate of the panels are supposed to be constant (during their useful life), due to the series-parallel configuration, the hazard rate of the PV Generator becomes time dependent.

Finally, by combing (8), (10) and (11)/(12), and on the basis of the actual system architecture and components, the complete expression for the Reliability of the whole PV System $R_{P V_{-} \text {System }}(t)$ can be finally obtained.

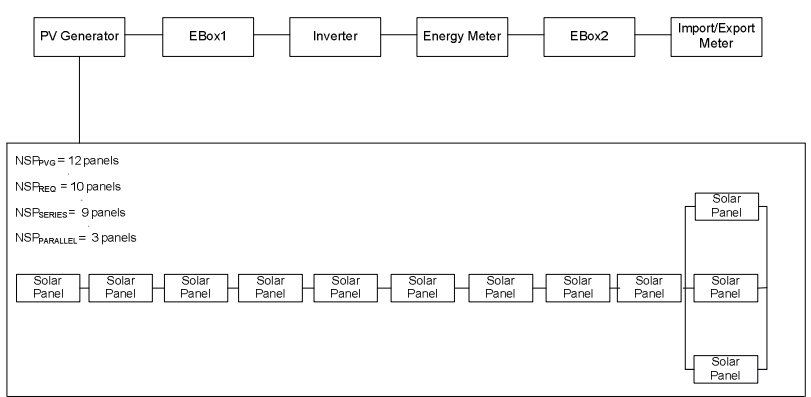

Fig. 2. The $R D B$ of a Residential PV System

\section{SERA, a domain-specific Software Environment for Reliability Analysis}

On the basis of the reliability model introduced in Section 3, SERA, a Java-based application which allows evaluating the reliability of residential PV systems, has been developed. Specifically, SERA allows the user to specify the architecture of the PV System under analysis in terms of the its componets and their characteristics (MTTF/hazard rate, electrical features, performances). Then, by applying the proposed series-parallel system reliability model, the Reliability Block Diagram (RDB) of the System is automatically generated and then System Reliability calculated with refererence to the time horizon specified by the user. When SERA is exploited during the System Design phase, on the basis of the reliability results, the user can modify the system architecture and/or the exploited components so to improve system reliability and/or evaluate different design choices; finally, a complete documentation which reports the performed reliability analysis are automatically generated.

As an example, Fig. 3 reports some screenshots of the current version of SERA which are referred to the reliability analysis of a PV System whose RDB is that of Fig. 2 and whose constant components failure rates $\left(\lambda_{\text {comp }}(t)=\lambda_{\text {comp }}=1 / M T T F_{\text {comp }}\right)$ are reported in Table II (in failures per hours). With a time horizon of 10.000 hours the system reliability calculated by SERA is equals to 0,206 , i.e. there is a probability of $20,6 \%$ that the system will operate for 10.000 without any failures or, in other words, a probability of $79,4 \%$ that the system fails within 10.000 hours. 


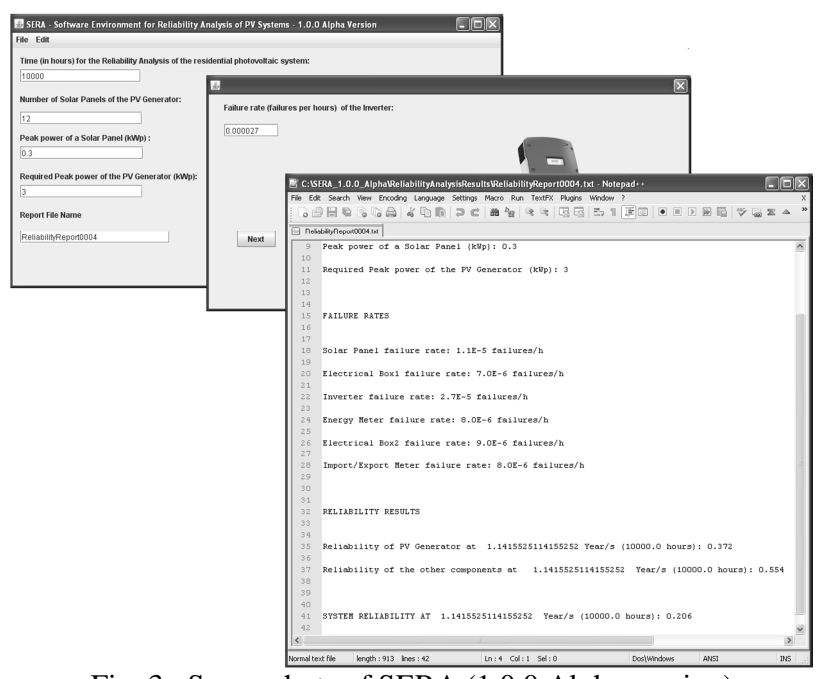

Fig. 3. Screenshots of SERA (1.0.0 Alpha version)

Table II. - Failure rates of the example PV system

\begin{tabular}{|l|c|}
\hline \multicolumn{1}{|c|}{ Component } & $\begin{array}{c}\lambda \\
\text { (failures per hours) }\end{array}$ \\
\hline Solar Panel & 0,000011 \\
\hline EBox1 & 0,000007 \\
\hline Inverter & 0,000027 \\
\hline Energy Meter & 0,000008 \\
\hline EBox2 & 0,000009 \\
\hline Import/Export Meter & 0,000008 \\
\hline
\end{tabular}

By comparing the reliability results obtained from SERA with the empirical data of 30 real PV system installations, the difference between the calculated $R_{P V_{-} S Y S T E M}(t)$ and the observed system reliability ranges from $4 \%$ to $12 \%$, depending on the chosen time horizon, while its main value is about $6 \%$. Moreover, the reliability results obtained from SERA have been compared with those produced by applying the RDB model proposed in [6], which represents a notable research effort in the reliability analysis of solar power system. As the model proposed in [6] is not specifically conceived for residential PV systems a preliminary adaptation phase was necessary; however, the obtained results showed a general overestimation of system reliability respect both to the values calculated by SERA and those coming from the empirical data. These differences indicate that the empirical reliability of a system does not only depend on the system architecture and components but also on the quality of the system implementation and actual operating conditions; these factors should also be taken into account in an extension of the proposed reliability model.

Currently SERA is in the Alpha-testing phase and the 1.0.0 Beta Version is going to be released in December 2010 as an Open Source software under the LGPL (GNU Lesser General Public License) license. Specifically, the Alpha-testing is performed by SAFEM S.r.L. ${ }^{2}$, an Italian company with extensive experience in designing and implementing commercial, industrial and residential PV systems. In particular, in the design of new Residential PV Systems the reliability analysis performed by SERA is exploited by SAFEM to meet the reliability

\footnotetext{
${ }^{2}$ http://www.safem.net/
}

requirements and/or evaluate different design choices; moreover, the produced documentation is provided to customers to certify the goodness of the implemented PV solution and to allow programming/contracting an acceptable maintenance plan to keep the system operating effectively. For existing PV Systems a reliability analysis is performed to obtain useful indication for establishing a system maintenance schedule to communicate to customer.

\section{Conclusion}

The increasing adoption of PV systems, which are rapidly becoming the most diffused solution for residential installations, raises new and important issues related to their dependability. However, in their design an accurate dependability analysis is often missing manly due to the lack of specific methodological approaches and related ready-to-use tools. To overcome this lack, models and methods coming from the R.A.M.S. engineering discipline could be profitably exploited. To this aim, the paper presented a methodological approach for the reliability analysis of residential PV systems which is centered on the application of a series-parallel system reliability analysis model. Although the presented model refers to residential and grid-connected PV systems it can be easily extended and modified to perform the reliability analysis of commercial, industrial and residential, on-grid, off-grid and hybrid PV systems. To guide the user, in a step by step way, from the system architecture definition to the generation of the documentation which clearly reports the reliability analysis results, SERA, a Java-based application, has been developed and will be released in December 2010 under the LGPL license. Future research effort will be devoted to: (i) extend and/or modify the proposed model to perform the reliability analysis of commercial, industrial and residential, on-grid, off-grid and hybrid PV systems; (ii) enrich the proposed analysis model and technique by jointly exploiting other qualitative and quantitative reliability analysis techniques as Fault Tree Analysis and Markov Chain; (iii) integrate new defined models and techniques in the SERA analysis environment. Moreover, the next release of SERA will present an enriched, totally visual and more user-friendly GUI (Graphical User Interface) and will provide full support for the management of data concerning template of system architectures, components datasheet, and analysis results.

\section{Acknowledgement}

The authors wish to thank Dalila Masiello for the Alphatesting phase which she is performing at SAFERM S.r.L. and for her contribution in developing SERA.

\section{References}

[1] N. Apergisa, K. Menyahc, and Y. Wolde-Rufael, "On the causal dynamics between emissions, nuclear energy, renewable energy, and economic growth", Ecological Economics, Vol. 69, No. 11, pp. 2255-2260, Elsevier B.V., 2010. 
[2] S. J. Davis, K. Caldeira, and H. D. Matthews, "Future CO2 Emissions and Climate Change from Existing Energy Infrastructure", Science, Vol. 329, No. 5997, pp. 13301333, HighWire Press, 2010.

[3] P. Díaz1, M. A. Egido, and F. Nieuwenhout, "Dependability analysis of stand-alone photovoltaic systems", Progress in Photovoltaics: Research and Applications, Vol. 15, No. 3, pp. 245-264, John Wiley \& Sons Ltd., 2007.

[4] B. Dodson, D. Nolan, "Practical Reliability Engineering", John Wiley \& Sons Ltd., 2002.

[5] J. P. Dunlop, "Photovoltaic Systems", American Technical Publishers, 2009.

[6] L. Gan, G. S. Eskelanda, and H. H. Kolshus, "Green electricity market development: Lessons from Europe and the US", Energy Policy, Vol. 35, No. 1, pp. 144-155, Elsevier Ltd., 2007.

[7] D.L. Huffman, F. Antelme, "Availability analysis of a solar power system with graceful degradation", in Proc. of the Reliability and Maintainability Symposium, Fort Worth, TX, 26-29 January, 2009.

[8] P. Nemaa, R.K. Nemab, and S. Rangnekar, "A current and future state of art development of hybrid energy system using wind and PV-solar: A review", Renewable and Sustainable Energy Reviews, Vol. 13, No. 8, pp. 20962103, Elsevier Ltd., 2009.

[9] P. O'Connor, "Reliability Engineering Handbook (Quality and Reliability)", CRC Press, 1999. 